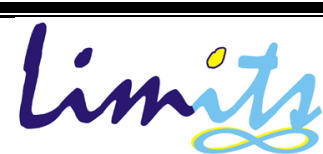

Limits: Journal of Mathematics and Its Applications

E-ISSN: 2579-8936

P-ISSN: 1829-605X

Vol. 18, No. 2, Nopember 2021, 169-186

DOI: http://dx.doi.org/10.12962/limits.v18i2.8751

\title{
Perbandingan Penyelesaian Integral Riemann, Lebesgue dan HK Berdasarkan Definisi
}

\author{
Miftahul Fikri ${ }^{1}{ }^{*}$, Samsurizal ${ }^{2}$, Andi Makkulau ${ }^{3}$ \\ 1,2,3 Jl. Lingkar Luar Barat, Duri Kosambi, Cengkareng, Jakarta 11750 \\ ${ }^{1,2,3}$ Fakultas Ketenagalistrikan dan Energi Terbarukan IT-PLN Jakarta Indonesia \\ e-mail:miftahul@itpln.ac.id
}

Diajukan: 25 Februari 2021, Diperbaiki: 17 Juni 2021, Diterima: 24 Juni 2021

\begin{abstract}
Abstrak
Sejak integral diperkenalkan oleh Newton dan Leibniz pada abad ke-17, alat ukur ini terus dilakukan kajian dan perumuman hingga saat ini. Terdapat tiga integral yang dikenal secara luas yaitu integral Riemann, integral Lebesgue dan integral HK. Pada masanya integral Riemann diterapkan untuk menyelesaikan berbagai persoalan tetapi kemudian diketahui memiliki keterbatasan. Integral Lebesgue muncul untuk mengatasi keterbatasan ini. Integral Lebesgue pun kemudian diketahui memiliki keterbatasannya tersendiri sehingga muncul integral Henstock-Kurzweil (integral HK) untuk mengatasi keterbatasan integral Lebesgue. Dalam banyak literatur seringkali untuk menyelesaikan soal integral tidak menggunakan definisi/ kerangka acuan yang sama sehingga sangat sulit memahami substansi maupun keterkaitan ketiga integral ini. Oleh karena itu, pada penelitian ini dilakukan perbandingan penyelesaian persoalan integral menggunakan definisi. Adapun dari empat persoalan integral yang dibahas, integral Riemann dapat menyelesaikan satu persoalan, integral Lebesgue dapat menyelesaikan dua persoalan dan integral HK dapat menyelesaikan seluruh persoalan yang dibahas.
\end{abstract}

Kata Kunci: integral Riemann, integral Lebesgue, integral Henstock-Kurzweil.

\begin{abstract}
Since the integral was introduced by Newton and Leibniz in the 17th century, this measuring instrument has continued to be examined and generalized today. There are three integrals that are widely known, namely the Riemann integral, the Lebesgue integral, and the HK integral. In his time the Riemann integral was applied to solve various problems, but it was later discovered to have limitations. Lebesgue integral emerges to overcome these limitations. Then the Lebesgue integral is also known to have its limitations so that the Henstock-Kurzweil integral (HK integral) emerges to overcome the limitations of the Lebesgue integral. In much literature, often solving integral problems does not use the definition / same frame of reference so that it is very difficult to understand the substance and the relationship between these three integrals. Therefore, this research was carried out the comparison of integral problem solving using definitions. As for the four problems of integral discussed, the Riemann integral can solve one problem, the Lebesgue integral can solve two problems and the HK integral can solve all the problems discussed.
\end{abstract}

Keywords: Riemann integral, Lebesgue integral, Henstock-Kurzweil integral

\section{Pendahuluan}

Sejak Newton dan Leibniz pada abad ke-17 mengkaji turunan dan integral secara terpisah, topik ini khususnya integral terus mengalami perkembangan hingga era modern [1]. Era ini dimulai pada tahun 1823 ketika Cauchy mendefinisikan integral fungsi kontinu pada selang tutup 
$[a, b]$ menggunakan konsep limit. Kemudian pada tahun 1854 Riemann melengkapi pekerjaan Cauchy dengan mendefinisikan integral fungsi terbatas pada interval $[a, b]$ [2] [3]. Integral Riemann memiliki banyak penerapan dalam berbagai metode dan permasalahan seperti transformasi Laplace [4], transformasi Fourier [5], persamaan diferensial [6], fuzzy [7], dan lainlain. Dalam perkembangannya ditemukan fungsi-fungsi takterintegralkan Riemann, berikut kekurangan integral Riemann [8]:

a. Jika suatu fungsi terintegralkan Riemann di interval terbatas $[a, b]$ dari garis real maka fungsi terbatas,

b. Terdapat turunan fungsi yang terbatas tidak terintegralkan Riemann,

c. Terdapat barisan fungsi $\left\{f_{n}\right\}_{n=1}^{\infty}$ seragam terbatas, fungsi terintegralkan Rieman konvergen titik demi titik ke fungsi $f$ di dalam interval terbatas $[a, b]$ dari garis real tetapi $f$ takterintegralkan Riemann di $[a, b]$.

Pada tahun 1902 Lebesgue melakukan perumuman untuk mengatasi tiga kekurangan pada integral Riemann tersebut [8]. Hingga saat ini, beberapa literatur masih melakukan kajian tentang integral Lebesgue seperti [9], [10], [11], [12]. Meskipun dapat mengatasi kekurangan integral Riemann, dalam kajian sifat-sifat lanjutan integral Lebesgue masih terdapat fungsi $F$ kontinu di [0,1] dengan $F^{\prime}$ tak terintegralkan Lebesgue di [0,1]. Pada tahun 1912, Denjoy mendefinisikan integral untuk menyelesaikan permasalahan ini. Kemudian pada tahun 1914, Oskar Perron melakukan pendekatan berbeda dengan mengutamakan aplikasi teori integralnya pada permasalahan persamaan diferensial dan potensial. Yang kemudian diketahui bahwa Integral Denjoy dan Perron adalah ekuivalen dan merupakan perumuman dari integral Newton, integral Riemann, dan integral Lebesgue [8] [13].

Meskipun integral Denjoy dan Perron cukup powerful akan tetapi diperlukan mempelajari teori matematika yang lebih banyak. Pada tahun 1957 Jaroslav Kurzweil mendefinisikan integral pada risetnya terkait persamaan diferensial, secara terpisah pada tahun 1968 Ralph Henstock medefinisikan integralnya [14]. Integral Kurzweil dan integral Henstok kemudian diketahui ekuivalen dan biasa disebut integral HK. Bukan hanya samapai disitu, ternyata integral HenstokKurzweil dan integral Denjoy-Perron juga ekuivalen [13]. Walaupun integral HK dan integral Denjoy-Perrron ekuivalen, Integral HK lebih banyak digunakan karena dalam pendefinisian dan perhitungannya seperti integral Riemann. Dalam banyak literatur, integral HK masih dilakukan kajian lebih lanjut seperti [15], [16], [17].

Dalam perhitungannya seperti [18], [19], [20], [12] untuk menyelesaikan permasalahan integral, baik integral Riemann, integral Lebesgue maupun integral HK seringkali tidak berdasarkan kerangka acuan yang sama (definisi) sehingga baik substansi maupun keterkaitan 
ketiga integral ini tidak dapat dilihat secara langsung. Oleh karena itu penelitian ini akan membahas penyelesaian integral Riemann, Lebesgue, dan HK pada beberapa soal integral berdasarkan definisi.

\section{Metode Penelitian}

Definisi 1 [[8][21]] Suatu partisi dari suatu interval $I=[a, b]$ adalah koleksi $P=\left\{I_{1}, I_{2}, \ldots, I_{n}\right\}$ dari non-overlapping interval tertutup yang mana gabungannya adalah $[a, b]$. Biasanya interval dilambangkan $I_{i}=\left[x_{i-1}, x_{i}\right]$, di mana

$$
a=x_{0}<\cdots<x_{i-1}<x_{i}<x_{n}=b .
$$

Titik-titik $x_{0}, x_{1}, \ldots, x_{n}$ adalah disebut titik-titik partisi dari P. Jika suatu titik $t_{i}$ dipilih dari setiap interval $I_{i}$, untuk $i=1,2, \ldots, n$, maka titik-titit $t_{i}$ disebut label-label dan himpunan pasangan terurut $\dot{P}=\left\{\left(I_{1}, t_{1}\right),\left(I_{2}, t_{2}\right), \ldots,\left(I_{n}, t_{n}\right)\right\}$ adalah disebut partisi label dari $I$.

Definisi 2 [[8][21]] Suatu gauge di dalam I adalah suatu fungsi strictly positifyang didefinisikan di dalam I. Jika $\delta$ adalah suatu gauge di dalam I, maka suatu (tagged) partisi $\dot{P}$ adalah dikatakan menjadi $\delta$ - fine jika $t_{i} \in I_{i} \subset\left[t_{i}-\delta\left(t_{i}\right), t_{i}+\delta\left(t_{i}\right)\right]$, untuk $i=1,2, \ldots, n$.

Definisi 3[[21]] Suatu fungsi $f:[a, b] \rightarrow \mathbb{R}$ adalah dikatakan terintegralkan Riemann di $[a, b]$ jika dipenuhi langkah-langkah/ pernyataan berikut:

1. Pilih $L \in \mathbb{R}$, ambil sembarang $\varepsilon>0$ dan pilih $\delta_{\varepsilon}>0$,

2. Ambil sembarang $n$ bilangan asli dan tetapkan sembarang koleksi dari titik-titik di dalam $[a, b]$, yaitu $\left\{x_{0}, x_{1}, x_{2}, \ldots, x_{n}\right\}$ sedemikian rupa sehingga $a=x_{0}<x_{1}<x_{2}<\ldots<$ $x_{n}=b$,

3. Tetapkan partisi $P=\left\{\left[x_{i-1}, x_{i}\right]\right\}_{i=1}^{n}$ subinterval dari $[a, b]$, ambil sembarang titik $x_{i}^{*} \in$ $\left[x_{i-1}, x_{i}\right]$ untuk $\mathrm{i}=1,2, \ldots \mathrm{n}$, hitung $\|P\|=\max \left\{x_{1}-x_{0}, x_{2}-x_{1}, \ldots, x_{n}-x_{n-1}\right\}$ dan tetapkan tag partisi $\dot{P}=\left\{\left[x_{i-1}, x_{i}\right], x_{i}^{*}\right\}_{i=1}^{n}$,

sedemikian rupa sehingga jika tag partisi $\dot{P}$ dari $[a, b]$ dengan $\|P\|<\delta_{\varepsilon}$ maka

$$
\left|\sum_{i=1}^{n} \boldsymbol{f}\left(\boldsymbol{x}_{\boldsymbol{i}}^{*}\right)\left(x_{i}-x_{i-1}\right)-L\right|<\varepsilon .
$$

Langkah-langkah/ pernyataan di atas merupakan pemaknaan dari definisi berikut: suatu fungsi $f:[a, b] \rightarrow \mathbb{R}$ adalah dikatakan terintegralkan Riemann di $[a, b]$ jika disini ada bilangan $L \in \mathbb{R} \exists$ $\forall \varepsilon>0$ disini ada $\delta_{\varepsilon}>0 \ni$ jika $\dot{P}$ adalah sembarang tag partisi dari $[a, b]$ dengan $\|\dot{P}\|<\delta_{\varepsilon}$, 
maka $\left|\sum_{i=1}^{n} \boldsymbol{f}\left(\boldsymbol{x}_{\boldsymbol{i}}^{*}\right)\left(x_{i}-x_{i-1}\right)-L\right|<\varepsilon$. Bilangan L jika ada disebut integral Riemann dari $f$ atas $[a, b]$ dan dilambangkan dengan $(R) \int_{[a, b]} f(x) d x$.

Definisi 4 [[22]] Suatu fungsi yang terukur dan terbatas $f: A \rightarrow \mathbb{R}$ adalah terintegralkan Lebesgue di $A$ jika dipenuhi langkah-langkah/ pernyataan berikut:

1. Pilih bilangan real $L$, ambil sembarang $\varepsilon>0$ dan pilih $\delta_{\varepsilon}>0$,

2. Tetapkan batas bawah terbesar $l=\inf \{f(x) \mid x \in A\}$ dan batas atas terkecil $u=1+$ $\sup \{f(x) \mid x \in A\} \quad(l, u$ dijamin keberadaannya karena $f$ terbatas), kemudian ambil sembarang $\mathrm{n}$ bilangan asli dan tetapkan sembarang koleksi dari titik-titik $\left\{y_{0}, y_{1}, \ldots, y_{n}\right\}$ sedemikian rupa sehingga $l=y_{0}<y_{1}<y_{2}<\ldots<y_{n}=u$,

3. Tetapkan partisi $P=\left\{\left[y_{i-1}, y_{i}\right]\right\}_{i=1}^{n}$ subinterval dari $[l, u]$, ambil sembarang titik $y_{i}^{*} \in$ $\left[y_{i-1}, y_{i}\right]$ untuk $i=1,2, \ldots n$, hitung $\|P\|=\max \left\{y_{1}-y_{0}, y_{2}-y_{1}, \ldots, y_{n}-y_{n-1}\right\}$ dan tetapkan tag partisi $\dot{P}=\left\{\left[y_{i-1}, y_{i}\right], y_{i}^{*}\right\}_{i=1}^{n}$,

sedemikian rupa sehingga jika tag partisi $\dot{P}$ dengan $\|P\|<\delta_{\varepsilon}$ dan m ukuran Lebesgue maka

$$
\left|\sum_{i=1}^{n} \boldsymbol{y}_{\boldsymbol{i}}^{*} \boldsymbol{m}\left(\left\{x \in A \mid \boldsymbol{y}_{\boldsymbol{i}-1} \leq \boldsymbol{f}(\boldsymbol{x})<\boldsymbol{y}_{\boldsymbol{i}}\right\}\right)-L\right|<\varepsilon .
$$

Langkah-langkah/ pernyataan di atas merupakan pemaknaan dari definisi berikut: suatu fungsi yang terukur dan terbatas $f: A \rightarrow \mathbb{R}$ adalah terintegralkan Lebesgue di A jika ada bilangan real $L \ni \forall \varepsilon>0 \exists \delta>0 \ni$ jika $\|P\|<\delta$ maka $\left|\sum_{\boldsymbol{i}=\mathbf{1}}^{\boldsymbol{n}} \boldsymbol{y}_{\boldsymbol{i}}^{*} \boldsymbol{m}\left(\left\{x \in A \mid \boldsymbol{y}_{\boldsymbol{i}-\mathbf{1}} \leq \boldsymbol{f}(\boldsymbol{x})<\boldsymbol{y}_{\boldsymbol{i}}\right\}\right)-L\right|<\varepsilon$. Bilangan L jika ada disebut integral Lebesgue dari $f$ atas $A$ dan dilambangkan dengan (L) $\int_{A} f d m$.

Definisi 5 [[8]] Suatu fungsi $f: I \rightarrow \mathbb{R}$ dengan $I$ interval tertutup di $R^{*}$ adalah dikatakan terintegralkan HK di I jika dipenuhi langkah-langkah/ pernyataan berikut:

1. Pilih $L \in \mathbb{R}$, ambil sembarang $\varepsilon>0$, pilih gauge $\delta_{\varepsilon}$ di $I$,

2. Ambil sembarang $\mathrm{n}$ bilangan asli dan tetapkan sembarang koleksi dari titik-titik di dalam $I$ yaitu $\left\{x_{0}, x_{1}, x_{2}, \ldots, x_{n}\right\}$ sedemikian rupa sehingga $x_{0}<x_{1}<x_{2}<\ldots<x_{n}$ dengan $x_{0}$ dan $x_{n}$ masing-masing batas bawah dan batas atas di $I$,

3. Tetapkan partisi $P=\left\{\left[x_{i-1}, x_{i}\right]\right\}_{i=1}^{n}$ subinterval dari $I$, ambil sembarang titik $x_{i}^{*} \in$ $\left[x_{i-1}, x_{i}\right]$ untuk $i=1,2, \ldots n$, hitung $\|P\|=\max \left\{x_{1}-x_{0}, x_{2}-x_{1}, \ldots, x_{n}-x_{n-1}\right\}$ dan tetapkan tag partisi $\dot{P}=\left\{\left[x_{i-1}, x_{i}\right], x_{i}^{*}\right\}_{i=1}^{n}$,

sedemikian rupa sehingga jika tag partisi $\dot{P}$ adalah $\delta_{\varepsilon}-$ fine dari $I$ maka 


$$
\left|\sum_{i=1}^{n} \boldsymbol{f}\left(\boldsymbol{x}_{\boldsymbol{i}}^{*}\right)\left(x_{i}-x_{i-1}\right)-L\right|<\varepsilon,
$$

Langkah-langkah/ pernyataan di atas merupakan pemaknaan dari definisi berikut: suatu fungsi $f: I \rightarrow \mathbb{R}$ dengan I interval tertutup di $R^{*}$ adalah dikatakan terintegralkan $H K$ di I jika $\exists L \in \mathbb{R} \exists$ $\forall \boldsymbol{\varepsilon}>\mathbf{0} \exists$ gauge $\delta_{\varepsilon}$ di I sedemikian rupa sehingga jika $\dot{P}$ adalah sembarang partisi $\delta_{\varepsilon}-$ fine dari I, maka $\left|\sum_{\boldsymbol{i}=\mathbf{1}}^{\boldsymbol{n}} \boldsymbol{f}\left(\boldsymbol{x}_{\boldsymbol{i}}^{*}\right)\left(x_{i}-x_{i-1}\right)-L\right|<\varepsilon$. Bilangan L jika ada disebut integral HK dari $f$ atas I dan dilambangkan dengan $(H K) \int_{I} f(x) d x$.

\section{Hasil dan Pembahasan}

Pada penelitiain ini akan mengkaji integral Riemann, integral Lebesque dan integral HK menggunakan definisi untuk menyelesaikan empat masalah integral sebagai berikut:

1. fungsi $f(x)=x$ di $[0,1]$,

2. fungsi Dirichlet di $[0,1]$,

3. fungsi $f(x)=\frac{1}{x^{2}}$ di $[1, \infty)$,

4. fungsi $f^{\prime}(x)$ di $[0,1]$ dengan $f(x)=\left\{\begin{array}{lc}0 & x=0 \\ x^{2} \cos \frac{\pi}{x^{2}}, & 0<x \leq 1\end{array}\right.$.

Masing-masing soal akan dibahas sebagai berikut:

1. Fungsi $f(x)=x$ di $[0,1]$.

a. Integral Riemann [23]

1. Pilih $L=\frac{1}{2}$, ambil sembarang $\varepsilon>0$ dan pilih $\delta_{\varepsilon}=\varepsilon$,

2. Ambil sembarang $\mathrm{n}$ bilangan asli dan tetapkan sembarang koleksi dari titik-titik di dalam $[0,1]$, yaitu $\left\{x_{0}, x_{1}, x_{2}, \ldots, x_{n}\right\}$ sedemikian rupa sehingga $0=x_{0}<x_{1}<$ $x_{2}<\ldots<x_{n}=1$,

3. Tetapkan partisi $P=\left\{\left[x_{i-1}, x_{i}\right]\right\}_{i=1}^{n}$ subinterval dari $[0,1]$, ambil sembarang titik $x_{i}^{*} \in$ $\left[x_{i-1}, x_{i}\right]$ untuk $i=1,2, \ldots n$, hitung $\|P\|=\max \left\{x_{1}-x_{0}, x_{2}-x_{1}, \ldots, x_{n}-x_{n-1}\right\}$ dan tetapkan tag partisi $\dot{P}=\left\{\left[x_{i-1}, x_{i}\right], x_{i}^{*}\right\}_{i=1}^{n}$,

Sedemikian rupa sehingga jika tag partisi $\dot{P}$ dari $[0,1]$ dengan $\|P\|<\delta_{\varepsilon}$ maka

$$
\begin{aligned}
\left|\sum_{i=1}^{n} f\left(x_{i}^{*}\right)\left(x_{i}-x_{i-1}\right)-L\right| & =\left|\sum_{i=1}^{n} x_{i}^{*}\left(x_{i}-x_{i-1}\right)-\frac{1}{2}\right| \\
= & \left|\sum_{i=1}^{n} x_{i}^{*}\left(x_{i}-x_{i-1}\right)-\overline{x_{l}}\left(x_{i}-x_{i-1}\right)+\overline{x_{l}}\left(x_{i}-x_{i-1}\right)-\frac{1}{2}\right|
\end{aligned}
$$




$$
=\left|\sum_{i=1}^{n}\left(x_{i}^{*}-\bar{x}_{l}\right)\left(x_{i}-x_{i-1}\right)+\sum_{i=1}^{n} \bar{x}_{l}\left(x_{i}-x_{i-1}\right)-\frac{1}{2}\right|
$$

di mana $\overline{x_{l}}=\frac{\left(x_{i}+x_{i-1}\right)}{2}$, untuk $i=1,2, \ldots, n$. Selanjutnya di dalam persamaan (1) diperoleh

$$
\begin{aligned}
\sum_{i=1}^{n} \bar{x}_{l}\left(x_{i}-x_{i-1}\right)-\frac{1}{2} & =\sum_{i=1}^{n} \frac{\left(x_{i}+x_{i-1}\right)}{2}\left(x_{i}-x_{i-1}\right)-\frac{1}{2} \\
& =\sum_{i=1}^{n} \frac{\left(x_{i}^{2}-x_{i-1}^{2}\right)}{2}-\frac{1}{2} \\
& =\frac{\left(x_{n}^{2}-x_{0}^{2}\right)}{2}-\frac{1}{2} \\
& =\frac{1}{2}-\frac{1}{2} \\
& =0
\end{aligned}
$$

Sehingga Persamaan (1) menjadi $\left|\sum_{i=1}^{n}\left(x_{i}^{*}-\bar{x}_{l}\right)\left(x_{i}-x_{i-1}\right)\right|$. Akibatnya,

$$
\begin{aligned}
\left|\sum_{i=1}^{n} f\left(x_{i}^{*}\right)\left(x_{i}-x_{i-1}\right)-\frac{1}{2}\right| & =\left|\sum_{i=1}^{n}\left(x_{i}^{*}-\bar{x}_{l}\right)\left(x_{i}-x_{i-1}\right)\right| \\
& \leq \sum_{i=1}^{n}\left|x_{i}^{*}-\bar{x}_{l}\right|\left(x_{i}-x_{i-1}\right) \\
& <\sum_{i=1}^{n} \varepsilon\left(x_{i}-x_{i-1}\right) \\
& =\varepsilon \sum_{i=1}^{n}\left(x_{i}-x_{i-1}\right) \\
& =\varepsilon .1 \\
& =\varepsilon .
\end{aligned}
$$

Sehingga $f(x)=x$ terintegralkan Riemann di $[0,1]$ dan $(R) \int_{[a, b]} x d x=\frac{1}{2}$.

b. Integral Lebesgue

1. Pilih bilangan real $L=\frac{1}{2}$, ambil sembarang $\varepsilon>0$ dan pilih $\delta_{\varepsilon}=\varepsilon$,

2. Tetapkan $l=\inf \{f(x) \mid x \in A\}=0$ dan $u=1+\sup \{f(x) \mid x \in A\}=2$, kemudian ambil sembarang $n>1$ bilangan asli dan tetapkan sembarang koleksi dari titik-titik $\left\{y_{0}, y_{1}, \ldots, y_{n}\right\}$ sedemikian rupa sehingga $0=y_{0}<y_{1}<y_{2}<\ldots<y_{n}=2$, 
3. Tetapkan partisi $P=\left\{\left[y_{i-1}, y_{i}\right]\right\}_{i=1}^{n}$ subinterval dari $[0,2]$, ambil sembarang titik $y_{i}^{*} \in$ $\left[y_{i-1}, y_{i}\right]$ untuk $i=1,2, \ldots n$, hitung $\|P\|=\max \left\{y_{1}-y_{0}, y_{2}-y_{1}, \ldots, y_{n}-y_{n-1}\right\}$ dan tetapkan tag partisi $\dot{P}=\left\{\left[y_{i-1}, y_{i}\right], y_{i}^{*}\right\}_{i=1}^{n}$,

sedemikian rupa sehingga jika tag partisi $\dot{P}$ dengan $\|P\|<\delta_{\varepsilon}$ dan $m$ ukuran Lebesgue maka

$$
\begin{aligned}
\left|\sum_{i=1}^{n} y_{i}^{*} \boldsymbol{m}\left(\left\{x \in[0,1] \mid y_{i-1} \leq f(x)<y_{i}\right\}\right)-L\right| \\
=\left|\sum_{i=1}^{r} y_{i}^{*} \boldsymbol{m}\left(\left\{x \in[0,1] \mid y_{i-1} \leq f(x)<y_{i}\right\}\right)-L\right| \\
+\left|\sum_{i=r+1}^{n} y_{i}^{*} \boldsymbol{m}\left(\left\{x \in[0,1] \mid y_{i-1} \leq f(x)<y_{i}\right\}\right)-L\right|
\end{aligned}
$$

di mana $\boldsymbol{y}_{r}^{*} \in\left\{y_{0}, y_{1}, \ldots, y_{n}\right\}$ bilangan terkecil yang lebih dari 1 akibatnya $\left|\sum_{i=r+1}^{n} \boldsymbol{y}_{\boldsymbol{i}}^{*} \boldsymbol{m}\left(\left\{x \in[0,1] \mid \boldsymbol{y}_{\boldsymbol{i}-\mathbf{1}} \leq \boldsymbol{f}(\boldsymbol{x})<\boldsymbol{y}_{\boldsymbol{i}}\right\}\right)-L\right|=0$, sehingga Persamaan menjadi

$$
\begin{aligned}
& \left|\sum_{i=1}^{n} y_{i}^{*} \boldsymbol{m}\left(\left\{x \in[0,1] \mid y_{i-1} \leq f(x)<y_{i}\right\}\right)-L\right| \\
& =\left|\sum_{i=1}^{r} y_{i}^{*} \boldsymbol{m}\left(\left\{x \in[0,1] \mid y_{i-1} \leq \boldsymbol{f}(x)<y_{i}\right\}\right)-L\right| \\
& =\left|\sum_{i=1}^{r} y_{i}^{*} \boldsymbol{m}\left(\left\{x \in[0,1] \mid y_{i-1} \leq f(x)<y_{i}\right\}\right)-\frac{1}{2}\right| \text {. } \\
& =\left|\sum_{i=1}^{r} x_{i}^{*}\left(x_{i}-x_{i-1}\right)-\frac{1}{2}\right|, \quad \text { di mana } \boldsymbol{x}_{\boldsymbol{i}}^{*}=\boldsymbol{f}^{-\mathbf{1}}\left(\boldsymbol{y}_{\boldsymbol{i}}^{*}\right) \\
& =\left|\sum_{i=1}^{n} x_{i}^{*}\left(x_{i}-x_{i-1}\right)-\bar{x}_{l}\left(x_{i}-x_{i-1}\right)+\bar{x}_{\imath}\left(x_{i}-x_{i-1}\right)-\frac{1}{2}\right| \\
& =\left|\sum_{i=1}^{n}\left(x_{i}^{*}-\bar{x}_{l}\right)\left(x_{i}-x_{i-1}\right)+\sum_{i=1}^{n} \bar{x}_{l}\left(x_{i}-x_{i-1}\right)-\frac{1}{2}\right|
\end{aligned}
$$

di mana $\overline{x_{l}}=\frac{\left(x_{i}+x_{i-1}\right)}{2}$, untuk $i=1,2, \ldots, n$. Selanjutnya di dalam Persamaan (3) diperoleh

$$
\begin{aligned}
\sum_{i=1}^{n} \bar{x}_{l}\left(x_{i}-x_{i-1}\right)-\frac{1}{2} & =\sum_{i=1}^{n} \frac{\left(x_{i}+x_{i-1}\right)}{2}\left(x_{i}-x_{i-1}\right)-\frac{1}{2} \\
& =\sum_{i=1}^{n} \frac{\left(x_{i}^{2}-x_{i-1}^{2}\right)}{2}-\frac{1}{2}
\end{aligned}
$$




$$
\begin{aligned}
& =\frac{\left(x_{n}^{2}-x_{0}^{2}\right)}{2}-\frac{1}{2} \\
& =\frac{1}{2}-\frac{1}{2} \\
& =0 .
\end{aligned}
$$

Sehingga Persamaan (3) menjadi $\left|\sum_{i=1}^{n}\left(x_{i}^{*}-\bar{x}_{l}\right)\left(x_{i}-x_{i-1}\right)\right|$. Akibatnya,

$$
\begin{aligned}
\left|\sum_{i=1}^{n} f\left(x_{i}^{*}\right)\left(x_{i}-x_{i-1}\right)-\frac{1}{2}\right| & =\left|\sum_{i=1}^{n}\left(x_{i}^{*}-\overline{x_{l}}\right)\left(x_{i}-x_{i-1}\right)\right| \\
& \leq \sum_{i=1}^{n}\left|x_{i}^{*}-\bar{x}_{l}\right|\left(x_{i}-x_{i-1}\right) \\
& <\sum_{i=1}^{n} \varepsilon \Delta x_{i} \\
& =\varepsilon \sum_{i=1}^{n}\left(x_{i}-x_{i-1}\right) \\
& =\varepsilon .1 \\
& =\varepsilon .
\end{aligned}
$$

Sehingga $f(x)=x$ terintegralkan Lebesgue di $[0,1]$ dan $(L) \int_{[0,1]} x d m=\frac{1}{2}$.

c. Integral HK

1. Pilih $L=\frac{1}{2}$, ambil sembarang $\boldsymbol{\varepsilon}>\mathbf{0}$ dan pilih gauge $\delta_{\varepsilon}=\varepsilon / 2$,

2. Ambil sembarang $\mathrm{n}$ bilangan asli dan tetapkan sembarang koleksi dari titik-titik di dalam $[0,1]$, yaitu $\left\{x_{0}, x_{1}, x_{2}, \ldots, x_{n}\right\}$ sedemikian rupa sehingga $0=x_{0}<x_{1}<$ $x_{2}<\ldots<x_{n}=1$,

3. Tetapkan partisi $P=\left\{\left[x_{i-1}, x_{i}\right]\right\}_{i=1}^{n}$ subinterval dari $[0,1]$, ambil sembarang titik $x_{i}^{*} \in$ $\left[x_{i-1}, x_{i}\right]$ untuk $i=1,2, \ldots n$, hitung $\|P\|=\max \left\{x_{1}-x_{0}, x_{2}-x_{1}, \ldots, x_{n}-x_{n-1}\right\}$ dan tetapkan tag partisi $\dot{P}=\left\{\left[x_{i-1}, x_{i}\right], x_{i}^{*}\right\}_{i=1}^{n}$,

Sedemikian rupa sehingga jika tag partisi $\dot{P}$ adalah $\delta_{\varepsilon}$ - fine dari $[0,1]$ maka

$$
\begin{aligned}
\left|\sum_{i=1}^{n} f\left(x_{i}^{*}\right)\left(x_{i}-x_{i-1}\right)-L\right| & =\left|\sum_{i=1}^{n} x_{i}^{*}\left(x_{i}-x_{i-1}\right)-\frac{1}{2}\right| \\
& =\left|\sum_{i=1}^{n} x_{i}^{*}\left(x_{i}-x_{i-1}\right)-\bar{x}_{l}\left(x_{i}-x_{i-1}\right)+\bar{x}_{l}\left(x_{i}-x_{i-1}\right)-\frac{1}{2}\right| \\
& =\left|\sum_{i=1}^{n}\left(x_{i}^{*}-\bar{x}_{l}\right)\left(x_{i}-x_{i-1}\right)+\sum_{i=1}^{n} \bar{x}_{l}\left(x_{i}-x_{i-1}\right)-\frac{1}{2}\right|
\end{aligned}
$$


di mana $\overline{x_{l}}=\frac{\left(x_{i}+x_{i-1}\right)}{2}$, untuk $i=1,2, \ldots, n$. Selanjutnya di dalam Persamaan (4) diperoleh

$$
\begin{aligned}
\sum_{i=1}^{n} \bar{x}_{l}\left(x_{i}-x_{i-1}\right)-\frac{1}{2} & =\sum_{i=1}^{n} \frac{\left(x_{i}+x_{i-1}\right)}{2}\left(x_{i}-x_{i-1}\right)-\frac{1}{2} \\
& =\sum_{i=1}^{n} \frac{\left(x_{i}^{2}-x_{i-1}^{2}\right)}{2}-\frac{1}{2} \\
& =\frac{\left(x_{n}^{2}-x_{0}^{2}\right)}{2}-\frac{1}{2} \\
& =\frac{1}{2}-\frac{1}{2} \\
& =0 .
\end{aligned}
$$

Sehingga Persamaan (4) menjadi $\left|\sum_{i=1}^{n}\left(x_{i}^{*}-\bar{x}_{l}\right)\left(x_{i}-x_{i-1}\right)\right|$. Akibatnya,

$$
\begin{aligned}
\mid \sum_{i=1}^{n} f\left(x_{i}^{*}\right)\left(x_{i}-x_{i-1}\right) & -\frac{1}{2}|=| \sum_{i=1}^{n}\left(x_{i}^{*}-\bar{x}_{l}\right)\left(x_{i}-x_{i-1}\right) \mid \\
& \leq \sum_{i=1}^{n}\left|x_{i}^{*}-\bar{x}_{l}\right|\left(x_{i}-x_{i-1}\right) \\
& <\sum_{i=1}^{n} \varepsilon \Delta x_{i} \\
& =\varepsilon \sum_{i=1}^{n}\left(x_{i}-x_{i-1}\right) \\
& =\varepsilon .
\end{aligned}
$$

Sehingga $f(x)=x$ terintegralkan HK di $[0,1]$ dan $(H K) \int_{[0,1]} f(x) d x=\frac{1}{2}$.

2. Fungsi Dirichlet di $[0,1]$. Misalkan $E$ himpunan bilangan rasional di $[0,1]$ dan $F$ himpunan bilangan irasional di $[0,1]$, definisikan fungsi $f$ di $[0,1]$ yaitu $f(x)=\left\{\begin{array}{ll}1, & x \in E \\ 0, & x \in F\end{array}\right.$.

a. Integral Riemann

1. Ambil sembarang $L \in \mathbb{R}$, pilih $\varepsilon>0$ dan $\varepsilon=\left\{\begin{array}{ll}<\max \{1-L, L-1\} ; & L \neq 1 \\ \{x \mid 0<x<1\} ; & L=1\end{array}\right.$, ambil sembarang $\delta>0$,

2. Tetapkan $r \in N$ dan $r>\max \left\{\left(\frac{1}{\delta}+1\right) \varepsilon, \frac{\varepsilon}{1-\varepsilon}\right\}$, tetapkan $n \in N, n \neq \frac{L r}{1-r}$ dan $n=$ $\left\{\begin{array}{ll}>\max \left\{\frac{-r(\varepsilon-L)}{\varepsilon-L+1}, \frac{-r(\varepsilon+L)}{\varepsilon+L-1}, \frac{1}{\delta}-r\right\} ; & L \neq 1 \\ \left\{x \mid \frac{1}{\delta}-r<x \leq \frac{-r(\varepsilon-1)}{\varepsilon}\right\} ; & L=1\end{array}\right.$, tetapkan $x_{0}=0, x_{i}=x_{i-1}+\frac{1}{n+r}$ untuk $i=1,2, \ldots, n+r$ sehingga diperoleh $0=x_{0}<x_{1}<x_{2}<\cdots<x_{n+r}=1$, 
3. Tetapkan $P=\left\{\left[x_{i-1}, x_{i}\right]\right\}_{i=1}^{n+r}, x_{i}^{*}=\frac{x_{i}+x_{i-1}}{2}$ untuk $i=1,2, \ldots, n+r$, hitung $\|P\|=$ $\frac{1}{n+r}$, tetapkan $\dot{P}=\left\{\left[x_{i-1}, x_{i}\right], x_{i}^{*}\right\}_{i=1}^{n+r}$,

sedemikian rupa sehingga $\|P\|=\frac{1}{n+r}<\delta$, maka

$$
\begin{aligned}
& \left|\sum_{i=1}^{n+r} f\left(x_{i}^{*}\right)\left(x_{i}-x_{i-1}\right)-L\right|=\left|\sum_{i=1}^{n} f\left(x_{i}^{*}\right)\left(x_{i}-x_{i-1}\right)+\sum_{i=n+1}^{n+r} f\left(x_{i}^{*}\right)\left(x_{i}-x_{i-1}\right)-L\right| \\
& =\left|\sum_{i=1}^{n} 1\left(x_{i}-x_{i-1}\right)+\sum_{i=n+1}^{n+r} 0\left(x_{i}-x_{i-1}\right)-L\right| \\
& =\left|\sum_{i=1}^{n}\left(x_{i}-x_{i-1}\right)-L\right| \\
& =\left|\sum_{i=1}^{n} \frac{1}{n+r}-L\right| \\
& =\left|\frac{n}{n+r}-L\right| \\
& \geq\left|\frac{\frac{-r(\varepsilon-L)}{\varepsilon-L+1}}{\frac{-r(\varepsilon-L)}{\varepsilon-L+1}+r}-L\right| \\
& \geq\left|\frac{\frac{-\frac{\varepsilon}{1-\varepsilon}(\varepsilon-L)}{\varepsilon-L+1}}{\frac{-\frac{\varepsilon}{1-\varepsilon}(\varepsilon-L)}{\varepsilon-L+1}+\frac{\varepsilon}{1-\varepsilon}}-L\right| \\
& =\left|\frac{\frac{-\varepsilon(\varepsilon-L)}{(1-\varepsilon)(\varepsilon-L+1)}}{\frac{-\varepsilon(\varepsilon-L)}{(1-\varepsilon)(\varepsilon-L+1)}+\frac{\varepsilon(\varepsilon-L+1)}{(1-\varepsilon)(\varepsilon-L+1)}}-L\right| \\
& =\left|\frac{\frac{-\varepsilon(\varepsilon-L)}{(1-\varepsilon)(\varepsilon-L+1)}}{\frac{\varepsilon}{(1-\varepsilon)(\varepsilon-L+1)}}-L\right| \\
& =\left|\frac{-\varepsilon(\varepsilon-L)}{(1-\varepsilon)(\varepsilon-L+1)} \frac{(1-\varepsilon)(\varepsilon-L+1)}{\varepsilon}-L\right| \\
& =|-\varepsilon+L-L| \\
& \geq \varepsilon \text {. }
\end{aligned}
$$

Sehingga fungsi Dirichlet di $[0,1]$ tak terintegralkan Riemann.

b. Integral Lebesgue

1. Pilih $L=0$, ambil sembarang $\varepsilon>0$ dan pilih $\delta_{\varepsilon} \leq \frac{\varepsilon}{2} \ni$, 
2. Tetapkan $l=\inf \{f(x) \mid x \in A\}=0$ dan $u=1+\sup \{f(x) \mid x \in A\}=2$, kemudian ambil sembarang $\mathrm{n}$ bilangan asli dan tetapkan sembarang koleksi dari titik-titik di $[0,2]$ sedemikian rupa sehingga $0=y_{0}<y_{1}<\cdots<y_{n}=2$,

3. Tetapkan partisi $P=\left\{\left[y_{i-1}, y_{i}\right]\right\}_{i=1}^{n}$ subinterval dari $[0,2]$, ambil sembarang titik $y_{i}^{*} \in$ $\left[y_{i-1}, y_{i}\right]$ untuk $i=1,2, \ldots n$, hitung $\|P\|=\max \left\{y_{1}-y_{0}, y_{2}-y_{1}, \ldots, y_{n}-y_{n-1}\right\}$ dan tetapkan tag partisi $\dot{P}=\left\{\left[y_{i-1}, y_{i}\right], y_{i}^{*}\right\}_{i=1}^{n}$.

Sedemikian rupa sehingga tag partisi $\dot{P}$ dengan $\|P\|<\delta_{\varepsilon}$ dan $m$ ukuran Lebesgue maka

$$
\begin{aligned}
& \left|\sum_{i=1}^{n} y_{i}^{*} \boldsymbol{m}\left(\left\{x \in A \mid y_{i-1} \leq f(x)<y_{i}\right\}\right)-L\right| \\
& =\left|\sum_{i=1}^{n} y_{i}^{*} \boldsymbol{m}\left(\left\{x \in A \mid \boldsymbol{y}_{i-1} \leq \boldsymbol{f}(\boldsymbol{x})<\boldsymbol{y}_{i}\right\}\right)-0\right| \\
& =\left|\sum_{i=1}^{n} y_{i}^{*} \boldsymbol{m}\left(\left\{x \in A \mid y_{i-1} \leq f(x)<y_{i}\right\}\right)\right| \\
& =\mid y_{1}^{*} \boldsymbol{m}\left(\left\{x \in A \mid y_{0} \leq f(x)<y_{1}\right\}\right) \\
& +\sum_{\substack{i=2 \\
i \neq j}}^{n} y_{i}^{*} \boldsymbol{m}\left(\left\{x \in A \mid y_{i-1} \leq f(x)<y_{i}\right\}\right) \\
& +y_{j}^{*} \boldsymbol{m}\left(\left\{x \in A \mid \boldsymbol{y}_{j-1} \leq f(x)=1<y_{j}\right\}\right) \\
& =\left|\boldsymbol{y}_{\mathbf{1}}^{*} \boldsymbol{m}\left(\left\{x \in A \mid \boldsymbol{y}_{\mathbf{0}} \leq \boldsymbol{f}(\boldsymbol{x})<\boldsymbol{y}_{\mathbf{1}}\right\}\right)+0+0\right| \\
& =\mid y_{1}^{*}\left(\boldsymbol{m}\left(\left\{x \in E \mid y_{0} \leq f(x)<y_{1}\right\}\right)\right. \\
& \left.+\boldsymbol{m}\left(\left\{x \in F \mid \boldsymbol{y}_{0} \leq \boldsymbol{f}(\boldsymbol{x})<\boldsymbol{y}_{1}\right\}\right)\right) \mid \\
& =\left|\boldsymbol{y}_{1}^{*} \boldsymbol{m}\left(\left\{x \in F \mid \boldsymbol{y}_{0} \leq \boldsymbol{f}(\boldsymbol{x})<\boldsymbol{y}_{1}\right\}\right)\right| \\
& =\left|y_{1}^{*}\left(y_{1}-y_{0}\right)\right| \\
& \leq\left|y_{1}^{*} \delta\right| \\
& <2 \delta \\
& <\varepsilon \text {. }
\end{aligned}
$$

Sehingga fungsi Dirichlet $f$ terintegralkan Lebesgue di $[0,1]$ dan $(L) \int_{[0,1]} f d m=0$. 
c. Integral HK [21]

1. Pilih $L=0$, ambil sembarang $\boldsymbol{\varepsilon}>\mathbf{0}, E$ terhitung sehingga dapat disimbolkan dengan $\left\{r_{k}\right\}_{k=1}^{\infty}$, definisikan $\delta_{\varepsilon}\left(r_{k}\right)=\frac{\varepsilon}{2^{k+2}}$ dan $\delta_{\varepsilon}(x)=1$ jika $x \in F$.

2. Ambil sembarang $\mathrm{n}$ bilangan asli dan tetapkan sembarang koleksi dari titik-titik di dalam $[0,1]$ yaitu $\left\{x_{0}, x_{1}, x_{2}, \ldots, x_{n}\right\}$ sedemikian rupa sehingga $0=x_{0}<x_{1}<x_{2}<$ $\ldots<x_{n}=1$,

3. Tetapkan partisi $P=\left\{\left[x_{i-1}, x_{i}\right]\right\}_{i=1}^{n}$ subinterval dari $[a, b]$, ambil sembarang titik $x_{i}^{*} \in$ $\left[x_{i-1}, x_{i}\right]$ untuk $i=1,2, \ldots n$, hitung $\|P\|=\max \left\{x_{1}-x_{0}, x_{2}-x_{1}, \ldots, x_{n}-x_{n-1}\right\}$ dan tetapkan tag partisi $\dot{P}=\left\{\left[x_{i-1}, x_{i}\right], x_{i}^{*}\right\}_{i=1}^{n}$,

sedemikian rupa sehingga jika tag partisi $\dot{P}=\left\{\left(I_{i}, x_{i}^{*}\right)\right\}_{i=1}^{n}$ adalah $\delta_{\varepsilon}$-fine dari $[0,1]$, maka

$$
\begin{aligned}
\left|\sum_{i=1}^{m+n} \boldsymbol{f}\left(\boldsymbol{x}_{\boldsymbol{i}}^{*}\right)\left(x_{i}-x_{i-1}\right)-L\right| & =\left|\sum_{i=1}^{\boldsymbol{m}+\boldsymbol{n}} \boldsymbol{f}\left(\boldsymbol{x}_{\boldsymbol{i}}^{*}\right)\left(x_{i}-x_{i-1}\right)-0\right| \\
& =\left|\sum_{i=1}^{\boldsymbol{m}+\boldsymbol{n}} \boldsymbol{f}\left(\boldsymbol{x}_{\boldsymbol{i}}^{*}\right)\left(x_{i}-x_{i-1}\right)\right| \\
& =\left|\sum_{i=1}^{\boldsymbol{m}} \boldsymbol{f}\left(\boldsymbol{x}_{\boldsymbol{i}}^{*}\right)\left(x_{i}-x_{i-1}\right)+\sum_{i=1}^{n} \boldsymbol{f}\left(\boldsymbol{x}_{\boldsymbol{i}}^{*}\right)\left(x_{i}-x_{i-1}\right)\right| \\
& =\left|\sum_{i=1}^{\boldsymbol{m}} \mathbf{0}\left(x_{i}-x_{i-1}\right)+\sum_{i=1}^{n} \mathbf{1}\left(x_{i}-x_{i-1}\right)\right| \\
& =\left|\sum_{i=1}^{n}\left(x_{i}-x_{i-1}\right)\right|
\end{aligned}
$$

Ambil $n \rightarrow \infty$, maka Persamaan (5) menjadi

$$
\begin{aligned}
& \leq\left|\sum_{i=1}^{n} 2 \delta_{\varepsilon}\left(t_{i}\right)\right| \\
& \leq\left|\sum_{i=1}^{n} 2 \frac{\varepsilon}{2^{k+2}}\right| \\
& \leq\left|\sum_{i=1}^{n} \frac{\varepsilon}{2^{k+1}}\right| \\
& =\left|\frac{\varepsilon}{2}\right| \\
& <\varepsilon .
\end{aligned}
$$

Sehingga fungsi Dirichlet $f$ terintegralkan HK di $[0,1]$ dan $(H K) \int_{[0,1]} f(x) d x=0$. 
3. Integral untuk fungsi $f(x)=\frac{1}{x^{2}}$ di $[1, \infty)$.

a. Integral Riemann

Karena domain fungsi tak terbatas, maka berdasarkan definisi fungsi tak terintegralkan.

b. Integral Lebesgue

Karena domain fungsi tak terbatas maka berdasarkan definisi, fungsi tak terintegralkan.

c. Integral HK [27]

1. Pilih $L=1$, ambil sembarang $\varepsilon>0$, definisikan gauge $\delta_{\varepsilon}$ yang terbatas di $[1, \infty]$ yaitu $\delta_{\varepsilon}(\infty)=\left(\frac{2}{\varepsilon}, \infty\right]$ dan $[w, \infty] \subset \delta_{\varepsilon}(\infty)$ maka

$$
\left|f(\infty) l([w, \infty))-\frac{1}{w}\right|=\frac{1}{w}<\frac{\varepsilon}{2}
$$

(Persamaan (6) dapat diartikan bahwa luas daerah di bawah fungsi $f$ atas interval $[w, \infty)$ harus bernilai $\left.\frac{1}{w}\right)$,

2. Ambil sembarang $n$ bilangan asli dan tetapkan sembarang koleksi dari titik-titik di dalam $I$ yaitu $\left\{x_{0}, x_{1}, x_{2}, \ldots, x_{n}\right\}$ sedemikian rupa sehingga $1=x_{0}<x_{1}<x_{2}<$ $\ldots<x_{n-1}<x_{n}=\infty$,

3. Tetapkan partisi $P=\left\{\left[x_{i-1}, x_{i}\right]\right\}_{i=1}^{n}$ subinterval dari $I$, ambil sembarang titik $x_{i}^{*} \in$ $\left[x_{i-1}, x_{i}\right]$ untuk $i=1,2, \ldots n$, hitung $\|P\|=\max \left\{x_{1}-x_{0}, x_{2}-x_{1}, \ldots, x_{n}-\right.$ $\left.x_{n-1}\right\}$ dan tetapkan tag partisi $\dot{P}=\left\{\left[x_{i-1}, x_{i}\right], x_{i}^{*}\right\}_{i=1}^{n}$,

sedemikian rupa sehingga jika tag partisi $\dot{P}$ adalah $\delta_{\varepsilon}-$ fine dari $[1, \infty]$ maka

$$
\begin{aligned}
\mid \sum_{i=1}^{n} \boldsymbol{f}\left(\boldsymbol{x}_{\boldsymbol{i}}^{*}\right) & \left(x_{i}-x_{i-1}\right)-1|=| f(\infty) l\left(I_{n}\right)+\sum_{i=1}^{n-1} \boldsymbol{f}\left(\boldsymbol{x}_{\boldsymbol{i}}^{*}\right)\left(x_{i}-x_{i-1}\right)-1 \mid \\
& =\left|f(\infty) l\left(I_{n}\right)-\frac{1}{x_{n-1}}+\frac{1}{x_{n-1}}+\sum_{i=1}^{n-1} f\left(x_{i}^{*}\right)\left(x_{i}-x_{i-1}\right)-1\right| \\
& \leq\left|f(\infty) l\left(I_{n}\right)-\frac{1}{x_{n-1}}\right|+\left|\sum_{i=1}^{n-1} \frac{1}{\left(x_{i}^{*}\right)^{2}}\left(x_{i}-x_{i-1}\right)+\frac{1}{x_{n-1}}-1\right| \\
& =\left|f(\infty) l\left(I_{n}\right)-\frac{1}{x_{n-1}}\right|+\left|\sum_{i=1}^{n-1} \frac{1}{\left(x_{i}^{*}\right)^{2}}\left(x_{i}-x_{i-1}\right)+\sum_{i=1}^{n-1}-\frac{1}{x_{i-1}}+\frac{1}{x_{i}}\right| \\
& =\left|f(\infty) l\left(I_{n}\right)-\frac{1}{x_{n-1}}\right|+\left|\sum_{i=1}^{n-1}\left(\frac{1}{\left(x_{i}^{*}\right)^{2}}\left(x_{i}-x_{i-1}\right)-\left(\frac{1}{x_{i-1}}-\frac{1}{x_{i}}\right)\right)\right| \\
& =\left|f(\infty) l\left(I_{n}\right)-\frac{1}{x_{n-1}}\right|+\sum_{i=1}^{n-1}\left|\frac{\left(x_{i-1} x_{i}-\left(x_{i}^{*}\right)^{2}\right)}{\left(x_{i}^{*}\right)^{2}}\right|\left|\frac{1}{x_{i-1}}-\frac{1}{x_{i}}\right|
\end{aligned}
$$




$$
\begin{aligned}
& \leq\left|f(\infty) l\left(I_{n}\right)-\frac{1}{x_{n-1}}\right|+\sum_{i=1}^{n-1} \frac{x_{i}-x_{i-1}}{x_{i}^{*}}\left(\frac{1}{x_{i-1}}-\frac{1}{x_{i}}\right) \\
& \leq \frac{\varepsilon}{2}+\sum_{i=1}^{n-1} \frac{x_{i}-x_{i-1}}{x_{i}^{*}}\left(\frac{1}{x_{i-1}}-\frac{1}{x_{i}}\right) .
\end{aligned}
$$

Definisikan $\delta_{\varepsilon}\left(x_{i}^{*}\right)=\left(x_{i}^{*}-\frac{\varepsilon x_{i}^{*}}{4}, x_{i}^{*}+\frac{\varepsilon x_{i}^{*}}{4}\right)$ sehingga

$$
\begin{aligned}
\left|\sum_{i=1}^{n} f\left(x_{i}^{*}\right)\left(x_{i}-x_{i-1}\right)-1\right| & \leq \frac{\varepsilon}{2}+\sum_{i=1}^{n-1} \frac{\varepsilon x_{i}^{*}}{2 x_{i}^{*}}\left(\frac{1}{x_{i-1}}-\frac{1}{x_{i}}\right) \\
& =\frac{\varepsilon}{2}+\frac{\varepsilon}{2} \sum_{i=1}^{n-1}\left(\frac{1}{x_{i-1}}-\frac{1}{x_{i}}\right) \\
& =\frac{\varepsilon}{2}+\frac{\varepsilon}{2}\left(1-\frac{1}{x_{n-1}}\right) \\
& <\varepsilon .
\end{aligned}
$$

Sehingga fungsi $f(x)=\frac{1}{x^{2}}$ terintegralkan $\operatorname{HK}$ di $[1, \infty)$ dan $(H K) \int_{[1, \infty)} f(x) d x=1$.

4. Integral $f^{\prime}$ jika diketahui $f(x)=\left\{\begin{array}{cc}0, & x=0 \\ x^{2} \cos \frac{\pi}{x^{2}}, & 0<x \leq 1\end{array}\right.$. Dengan menggunakan turunan perkalian fungsi akan diperoleh $f^{\prime}(x)=\left\{\begin{array}{lc}0, & x=0 \\ 2 x \cos \frac{\pi}{x^{2}}+\frac{2 \pi}{x} \sin \frac{\pi}{x^{2}}, & 0<x \leq 1\end{array}\right.$.

a. Integral Riemann

1. Ambil sembarang $L \in \mathbb{R}$, pilih $0<\varepsilon<2 \pi$, ambil sembarang $\delta>0$,

2. Tetapkan $n \in N$, tetapkan $x_{0}=0, x_{i}=x_{i-1}+\frac{1}{n}$ untuk $i=1,2, \ldots, n$ sehingga diperoleh $0=x_{0}<x_{1}<x_{2}<\cdots<x_{n}=1$,

3. Tetapkan $P=\left\{\left[x_{i-1}, x_{i}\right]\right\}_{i=1}^{n}, x_{i}^{*}=\frac{x_{i}+x_{i-1}}{2}$ untuk $i=1,2, \ldots, n$, hitung $\|P\|=\frac{1}{n}$, tetapkan $\dot{P}=\left\{\left[x_{i-1}, x_{i}\right], x_{i}^{*}\right\}_{i=1}^{n}$,

Sedemikian rupa sehingga $\|P\|=\frac{1}{n}<\delta$,

$$
\begin{aligned}
\left|\sum_{i=1}^{n} f^{\prime}\left(x_{i}^{*}\right)\left(x_{i}-x_{i-1}\right)-L\right| & =\left|f^{\prime}\left(x_{1}^{*}\right)\left(x_{1}-x_{0}\right)+\sum_{i=2}^{n} f^{\prime}\left(x_{i}^{*}\right)\left(x_{i}-x_{i-1}\right)-L\right| \\
& =\left|f^{\prime}\left(x_{1}^{*}\right)\left(x_{1}-x_{0}\right)-\left(-\sum_{i=2}^{n} f^{\prime}\left(x_{i}^{*}\right)\left(x_{i}-x_{i-1}\right)+L\right)\right| \\
& \geq\left|f^{\prime}\left(x_{1}^{*}\right)\left(x_{1}-x_{0}\right)\right|+\left|-\sum_{i=2}^{n} f^{\prime}\left(x_{i}^{*}\right)\left(x_{i}-x_{i-1}\right)+L\right|
\end{aligned}
$$




$$
\begin{aligned}
& \geq\left|f^{\prime}\left(x_{1}^{*}\right)\left(x_{1}-x_{0}\right)\right| \\
& =\left|f^{\prime}\left(\frac{1}{n}\right) \cdot\left(\frac{1}{n}\right)\right| \\
& =\left|\left(2 \frac{1}{n} \cos n^{2} \pi+2 \pi n \sin n^{2} \pi\right)\left(\frac{1}{n}\right)\right| \\
& =\left|\frac{2}{n^{2}} \cos n^{2} \pi+2 \pi \sin n^{2} \pi\right|
\end{aligned}
$$

Ketika $n \rightarrow \infty$ maka $\frac{2}{n^{2}} \cos n^{2} \pi=0$ dan $2 \pi \sin n^{2} \pi$ divergen direntang $[-2 \pi, 2 \pi]$. Akibatnya ada $n \ni\left|\sum_{i=1}^{n} f\left(x_{i}^{*}\right)\left(x_{i}-x_{i-1}\right)-L\right| \geq\left|2 \pi \sin n^{2} \pi\right| \geq \varepsilon$. Sehingga $f^{\prime}$ tak terintegralkan Riemann.

b. Integral Lebesgue

Karena $f^{\prime}$ tak terbatas di $[0,1]$, maka sesuai definisi tak terintegralkan Lebesgue.

c. Integral HK

1. Pilih $L=-1$, ambil sembarang $\boldsymbol{\varepsilon}>\mathbf{0}$, pilih gauge $\delta_{\varepsilon}$ di $[0,1]$,

2. Ambil sembarang $\mathrm{n}$ bilangan asli dan tetapkan sembarang koleksi dari titik-titik di dalam $[0,1]$ yaitu $\left\{x_{0}, x_{1}, x_{2}, \ldots, x_{n}\right\}$ sedemikian rupa sehingga $x_{0}<x_{1}<x_{2}<$ $\ldots<x_{n}$ dengan $x_{0}$ dan $x_{n}$ masing-masing batas bawah dan batas atas di $[0,1]$,

3. Tetapkan partisi $P=\left\{\left[x_{i-1}, x_{i}\right]\right\}_{i=1}^{n}$ subinterval dari $I$, ambil sembarang titik $x_{i}^{*} \in$ $\left[x_{i-1}, x_{i}\right]$ untuk $i=1,2, \ldots n$, hitung $\|P\|=\max \left\{x_{1}-x_{0}, x_{2}-x_{1}, \ldots, x_{n}-\right.$ $\left.x_{n-1}\right\}$ dan tetapkan tag partisi $\dot{P}=\left\{\left[x_{i-1}, x_{i}\right], x_{i}^{*}\right\}_{i=1}^{n}$,

sedemikian rupa sehingga jika tag partisi $\dot{P}$ adalah $\delta_{\varepsilon}-$ fine dari $[0,1]$. Ketika $f$ terdiferensialkan di $x_{i}^{*}$ maka disini ada $\delta_{\varepsilon}>0$ sedemikian rupa sehingga $x \in[0,1]$ jika $0<\left|x-x_{i}^{*}\right|<\delta_{\varepsilon}$ maka $\left|\frac{f(x)-f\left(x_{i}^{*}\right)}{x-x_{i}^{*}}-f^{\prime}\left(x_{i}^{*}\right)\right|<\varepsilon$. Sehingga,

$$
\begin{aligned}
\left|\sum_{i=1}^{n} f^{\prime}\left(x_{i}^{*}\right)\left(x_{i}-x_{i-1}\right)-L\right|=\left|\sum_{i=1}^{n} f^{\prime}\left(x_{i}^{*}\right)\left(x_{i}-x_{i-1}+x_{i}^{*}-x_{i}^{*}\right)+1\right| \\
=\left|\sum_{i=1}^{n} f^{\prime}\left(x_{i}^{*}\right)\left(x_{i}-x_{i-1}+x_{i}^{*}-x_{i}^{*}\right)-(-1-0)\right| \\
=\left|\sum_{i=1}^{n} f^{\prime}\left(x_{i}^{*}\right)\left(x_{i}-x_{i-1}+x_{i}^{*}-x_{i}^{*}\right)-(f(1)-f(0))\right| \\
=\left|\sum_{i=1}^{n} f^{\prime}\left(x_{i}^{*}\right)\left(x_{i}-x_{i-1}+x_{i}^{*}-x_{i}^{*}\right)-\left(\sum_{i=1}^{n} f\left(x_{i}\right)-f\left(x_{i-1}\right)\right)\right|
\end{aligned}
$$




$$
\begin{aligned}
& =\left|\sum_{i=1}^{n}\left(f^{\prime}\left(x_{i}^{*}\right)\left(x_{i}-x_{i-1}+x_{i}^{*}-x_{i}^{*}\right)-f\left(x_{i}\right)+f\left(x_{i-1}\right)+f\left(x_{i}^{*}\right)-f\left(x_{i}^{*}\right)\right)\right| \\
& \leq\left|\sum_{i=1}^{n} f^{\prime}\left(x_{i}^{*}\right)\left(x_{i}-x_{i}^{*}\right)-f\left(x_{i}\right)+f\left(x_{i}^{*}\right)\right| \\
& \quad+\left|\sum_{i=1}^{n} f^{\prime}\left(x_{i}^{*}\right)\left(x_{i}^{*}-x_{i-1}\right)-f\left(x_{i}^{*}\right)+f\left(x_{i-1}\right)\right| \\
& <\sum_{i=1}^{n} \varepsilon\left(x_{i}-x_{i}^{*}\right)+\sum_{i=1}^{n} \varepsilon\left(x_{i}^{*}-x_{i-1}\right) \\
& =\varepsilon \sum_{i=1}^{n}\left(x_{i}-x_{i-1}\right) \\
& =\varepsilon .
\end{aligned}
$$

\begin{tabular}{|c|c|c|c|}
\hline Integran & $\begin{array}{c}\text { Terintegralkan } \\
\text { Riemann }\end{array}$ & $\begin{array}{c}\text { Terintegralkan } \\
\text { Lebesgue }\end{array}$ & $\begin{array}{c}\text { Terintegralkan } \\
\text { HK }\end{array}$ \\
\hline$f(x)=x$ di $[0,1]$ & $\sqrt{ }$ & $\sqrt{ }$ & $\sqrt{ }$ \\
\hline$f(x)=\left\{\begin{array}{l}1 ; x \in \text { Bil rasional } \\
0 ; x \in \text { Bil irasional }\end{array} d i[0,1]\right.$ & $x$ & $\sqrt{ }$ & $\sqrt{ }$ \\
\hline$f(x)=\frac{1}{x^{2}} \operatorname{di}[1, \infty)$ & $x$ & $x$ & $\sqrt{ }$ \\
\hline$f^{\prime}(x)$ di $[0,1]$ jika $f(x)=\left\{\begin{array}{lr}0, & x=0 \\
x^{2} \cos \frac{\pi}{x^{2}}, & 0<x \leq 1\end{array}\right.$ & $x$ & $x$ & $\sqrt{ }$ \\
\hline
\end{tabular}

Berdasarkan pembahasan di atas, diperoleh hasil sebagai berikut:

Tabel 1. Integran dengan Integral Riemann, Lebesgue dan HK

\section{Simpulan}

Pada penelitiain ini membahas penyelesaian empat masalah integral (fungsi $f(x)=x$ di $[0,1]$, fungsi Dirichlet di $[0,1]$, fungsi $f(x)=\frac{1}{x^{2}}$ di $[1, \infty), f^{\prime}(x)$ di $[0,1]$ jika $f(x)=$ $\left\{\begin{array}{lc}0, & x=0 \\ x^{2} \cos \frac{\pi}{x^{2}}, & 0<x \leq 1\end{array}\right)$. Adapun hasil yang diperoleh sebagai berikut:

1. Berdasarkan definisi, integral Riemann hanya dapat menyelesaikan integral fungsi $f(x)=x$ di $[0,1]$.

2. Berdasarkan definisi, integral Lebesgue dapat menyelesaikan integral fungsi $f(x)=x$ di $[0,1]$ dan fungsi Dirichlet di $[0,1]$.

3. Berdasarkan definisi, integral HK mampu menyelesaikan seluruh permasalahan integral ini. 


\section{Daftar Pustaka}

[1] E. Cassirer, "Newton and Leibniz," Philos. Rev., vol. 52, no. 4, pp. 366-391, 1943.

[2] I. N. Pesin, Classical And Modern Integration Theories. ACADEMIC PRESS, 1970.

[3] P. Y. Lee and R. Vyborny, The integral an easy approach after Kurzweil and Henstock. Cambridge University Press, 2000.

[4] N. Anjum and J. H. He, "Laplace transform: Making the variational iteration method easier," Appl. Math. Lett., vol. 92, pp. 134-138, 2019.

[5] P. Borghesani, P. Pennacchi, S. Chatterton, and R. Ricci, "The velocity synchronous discrete Fourier transform for order tracking in the field of rotating machinery," Mech. Syst. Signal Process., vol. 44, no. 1-2, pp. 118-133, 2014.

[6] X. Zhang, L. Liu, Y. Wu, and Y. Zou, "Existence and uniqueness of solutions for systems of fractional differential equations with Riemann-Stieltjes integral boundary condition," Adv. Differ. Equations, vol. 2018, no. 1, 2018.

[7] F. Santo Pedro, E. Esmi, and L. C. de Barros, "Calculus for linearly correlated fuzzy function using Fréchet derivative and Riemann integral," Inf. Sci. (Ny)., vol. 512, no. 88882, pp. 219-237, 2020.

[8] L. T. Yeong, Henstock-Kurzweil Integration On Euclidean Spaces. World Scientific, 2011.

[9] D. Zeng, R. Zhang, S. Zhong, G. Yang, Y. Yu, and K. Shi, "Novel Lebesgue-integralbased approach to improved results for neural networks with additive time-varying delay components,” J. Franklin Inst., vol. 354, no. 16, pp. 7543-7565, 2017.

[10] E. Dubon and A. San Antolín, "The Lebesgue differentiation theorem revisited," Expo. Math., vol. 37, no. 3, pp. 322-332, 2019.

[11] S. Gudder, "Quantum measure and integration theory," J. Math. Phys., vol. 123509, 2009.

[12] F. Móricz, "The Lebesgue summability of trigonometric integrals," J. Math. Anal. Appl., vol. 390, no. 1, pp. 188-196, 2012.

[13] R. A. Gordon, The integrals of Lebesgue, Denjoy, Perron, and Henstock. 1994.

[14] R. Henstock, “A Riemann-Type Integral of Lebesgue Power,” Can. J. Math., vol. 20, no. X, pp. 79-87, 1968.

[15] G. Ye and W. Liu, "The distributional Henstock-Kurzweil integral and applications," Monatshefte fur Math., vol. 181, no. 4, pp. 975-989, 2016.

[16] J. Malý and W. F. Pfeffer, "Henstock-Kurzweil integral on BV sets," Math. Bohem., vol. 141, no. 2, pp. 217-237, 2016. 
[17] W. Liu, G. Ye, and D. Zhao, "The distributional Henstock-Kurzweil integral and applications II,” J. Nonlinear Sci. Appl., vol. 10, no. 1, pp. 290-298, 2017.

[18] A. Pruthi, "Riemann integral vs. Lebesgue integral: A perspective view," Adv. Math. Sci. $J$. , vol. 9, no. 7, pp. 4505-4512, 2020.

[19] W. Wojas and J. Krupa, "Familiarizing Students with Definition of Lebesgue Integral: Examples of Calculation Directly from Its Definition Using Mathematica," Math. Comput. Sci., vol. 11, no. 3-4, pp. 363-381, 2017.

[20] T. Kiria and G. Pantsulaia, "Calculation of Lebesgue integrals by using uniformly distributed sequences," Trans. A. Razmadze Math. Inst., vol. 170, no. 3, pp. 402-409, 2016.

[21] R. G. Bartle and Donald R. Sherbert, "Introduction to Real Analysis.” John Wiley \& Sons, p. 1015024, 2011.

[22] D. S. Kurtz and C. W. Swartz, Theories of Integration, vol. 13. World Scientific, 2012.

[23] R. P. Agarwal, C. Flaut, and D. O'Regan, An Introduction to Real Analysis. 2018.

[24] H. Wilcox and D. L. Myres, An Introduction to Lebesgue Integration and Fourier Series. ROBERT E. KRIEGER PUBLISHING COMPANY, INC., 1978.

[25] H. L. Royden. and P. M. Fitzpatrick, Real Analysis. Prentice Hall, 2010.

[26] R. G. Bartle, A Modern Theory of Integration. American Mathematical Society, 2001.

[27] C. Swartz, Gauge Integral. World Scientific, 2001. 